\title{
Nasal chondromesenchymal hamartoma, a rare pediatric tumor: Case report
}

\author{
Adnan Ünal ${ }^{1}$, Rauf Oğuzhan $\mathrm{Kum}^{2}$, Yonca $\mathrm{Avc1}^{2}$, Devrim Tuba Ünal ${ }^{3}$ \\ ${ }^{1}$ Department of Otolaryngology, Hitit University Faculty of Medicine, Çorum, Clinics of ${ }^{2}$ Otolaryngology and ${ }^{3}$ Pathology, \\ Ankara Numune Training and Research Hospital, Ankara, Turkey. E-mail: yoncavci@hotmail.com \\ Received: 3 June 2015, Revised: 13 July 2015, Accepted: 24 August 2015
}

SUMMARY: Ünal A, Kum RO, Avcı Y, Ünal DT. Nasal chondromesenchymal hamartoma, a rare pediatric tumor: case report. Turk J Pediatr 2016; 58: 208-211.

Nasal chondromesenchymal hamartoma has characteristic clinicopathological features and it is accepted as the upper airway analogue of mesenchymal hamartoma of the chest wall. It is a rare lesion and only 31 cases have been reported in the English literature until 2014. In this article, a 13-year-old nasal chondromesenchymal hamartoma case is presented, which is the first nasal chondromesenchymal hamartoma case from Turkey. Although, nasal chondromesenchymal hamartoma has been accepted as a benign lesion, the possibility of malignant transformation should be kept in mind, and detailed histologic examination should be performed particularly in adult nasal chondromesenchymal hamartoma cases.

Key words: chondromesenchymal hamartoma, nasal mass, pediatric.

Hamartoma is focal overgrowth of cells and tissues that are natural for the organ which they are included in. Even though cellular elements are mature, they do not give rise to a normal structure like surrounding tissues. Hamartomas can be considered as a connection between malformations and neoplasia ${ }^{1}$.

Mesenchymal hamartoma, also called mesenchymoma, has two or more mesenchymal elements in addition to fibrous tissue ${ }^{1}$. Mesenchymal hamartoma presents with mass in liver, eyelid and chest wall particularly in pediatric age group. If it is seen in chest wall, generally in neonates and infants, it is called "mesenchymal hamartoma of the chest wall"

Nasal chondromesenchymal hamartoma was first described by McDermott et al. ${ }^{2}$ in 1998. Nasal chondromesenchymal hamartoma has characteristic clinicopathological features, and it is accepted as upper airway analogue of mesenchymal hamartoma of the chest wall ${ }^{3}$. Nasal chondromesenchymal hamartoma is a rare lesion, and only 31 cases have been reported in the English literature until $2014^{2,4,5}$. In this article, a 13-year-old nasal chondromesenchymal hamartoma case is presented, which is the first case from Turkey.

\section{Case Report}

A 13-year-old female patient presented with nasal obstruction which had been present for 6 months. Endoscopic examination revealed a gray-white, locally hemorrhagic mass totally filling the left side of nasal cavity.

Computed tomography scan of paranasal sinuses showed a $33 \times 61 \mathrm{~mm}$ mass which originated from lateral nasal wall, extended to choana, and deviated the nasal septum towards right side. There was indentation in medial wall of left maxillary sinus and loss of aeration in left maxillary, frontal, and sphenoid sinuses (Fig. 1).

Contrast-enhanced maxillofacial magnetic resonance imaging showed a mass lesion filling left nasal cavity, extending to left frontal sinus and nasopharynx, and deviated nasal septum to right side. The mass was hypointense on T1A series, and hyperintense and heterogeneous on T2A series. It was contrast-enhanced after injection of intravenous contrast material was administered. The medial wall of orbit seemed destructed, and medial rectus muscle seemed pushed laterally by the mass, but apparent invasion was not seen (Fig. 2). 
The mass was biopsied in another hospital before patient admitted to our clinic. We learned that the pathologist suspected lipoblastoma and a mesenchymal tumor, but could not make a differential diagnosis. The paraffin blocks were brought to our hospital, and examined by the Pathology department of our hospital. A chondroid chordoma or a chondroid neoplasia with myxoid characteristics was considered based upon histological and immunohistochemical properties, but the final diagnosis could not be established. Endoscopic tumor resection was performed under general anesthesia. The mass, which filled the left nasal cavity, has fat, bone and cartilage like tissues inside. It was seen that the mass extended until the anterior wall of sphenoid sinus and pushed nasal septum to the right side. Nasopharynx was tumor free, and complete surgical resection was performed.

Pathology report revealed that the lesion was surrounded by microscopically partially reactive bony lamellae, and a nodular pattern was seen in the submucosal area. It was reported that the lesion had basophilic myxoid stroma, hyaline cartilage-like lobular areas, oval-spindle shaped, bipolar mesenchymal cells, which locally had lacunar arrangement. There were hyperchromatic nucleated cells in the lesion that also contained fibroblast-like cells in a fascicular manner. However, there was no remarkable mitotic activity or necrosis. On immunohistochemical examination, tumor cells were positive for CD34, vimentin, S100, EMA and GFAP. Ki67 proliferation index was about $10 \%$. When both histopathological radiological characteristics of the mass were taken into account, the histopathological diagnosis of the mass was reported as "nasal chondromesenchymal hamartoma” (Fig. 3-4).

In the postoperative period no adjuvant therapies were administered. There is no recurrence after 1 year postoperatively.

\section{Discussion}

Nasal chondromesenchymal hamartoma is a rare benign lesion which originates from nasal cavity and/ or paranasal sinuses, and it is usually seen in infants. In 1998, McDermott et al. ${ }^{2}$ described nasal chondromesenchymal hamartoma with mixed stromal and chondroid tissues at varying rates, and it was accepted as upper airway analogue of mesenchymal hamartoma of chest wall, as a new pathologic entity ${ }^{2,3}$. Beforehand, it was named as chondroid hamartoma, mesenchymoma and nasal hamartoma ${ }^{2}$. Initially it was thought that tumor was developmental or congenital in origin, but later adult case reports had confuted this hypothesis since those patients were asymptomatic during their childhood. Environmental and hormonal factors with an underlying genetic predisposition have been accused in its etiopathogenesis ${ }^{5,6}$.

DICER 1 is an RNAse endoribonuclease that plays a critical role in microRNA and RNAinterference functional pathways. Mutation in the DICER 1 gene increases the risk of developing various tumors ${ }^{7}$. Pleuropulmonary blastoma (PPB) is a rare pediatric tumor of the lungs and pleura, and there is evidence that the genetic pathogenesis of this benign tumor is similar to nasal chondromesenchymal hamartoma ${ }^{8}$. Stewart et al. ${ }^{8}$ found pathogenic germline DICER 1 mutations in $75 \%$ of patients who had nasal chondromesenchymal hamartoma and PPB. They also reported that 2 of 3 PPB patients with other nasal lesions including Schneiderian papilloma, chronic sinusitis with cysts and allergic nasal polyp with eosinophils had germline DICER 1 mutations. It should be kept in mind that nasal chondromesenchymal hamartoma can be a part of the DICER 1 tumor spectrum including PPB, cystic nephroma, and Wilms tumor, Sertoli-Leydig cell tumors, pineablastoma, botyroid type embryonal rhabdomyosarcaoma of uterine cervix.

Although nasal chondromesenchymal hamartoma is usually seen in infants and children, the oldest patient reported in literature is 69 years old 5 . Clinical features vary in relation with the size and localization of lesion, but rhinorrhea, epistaxis, dyspnea, feeding problems and ophthalmological symptoms can be seen ${ }^{6}$.

On histopathological examination, nasal chondromesenchymal hamartoma consists of focal lobulated various mesenchymal components. The predominating components are irregular, mature and immature hyaline cartilage islands. Those cartilage islands have distinct borders from the surrounding stromal tissue $2,5,6,9$. Similar to other case reports in the literature, our histopathological findings 


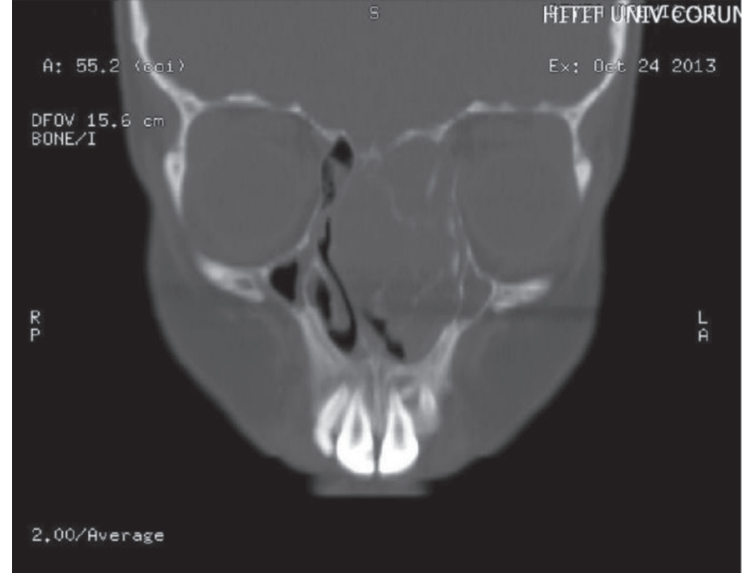

Fig. 1. Computed tomography scan of paranasal sinuses shows mass deviating the nasal septum towards right side

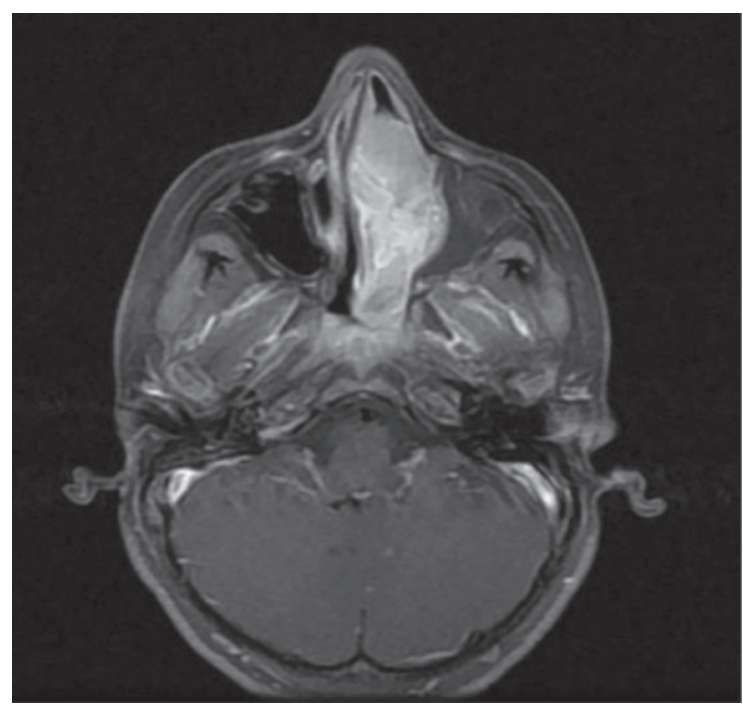

Fig. 2. Contrast-enhanced maxillofacial magnetic resonance imaging shows a mass lesion filling left nasal cavity on axial $\mathrm{T} 2$ weighted images

revealed a basophilic myxoid matrix, focal hyaline cartilage-looking areas, and oval-spindle shaped bipolar mesenchymal cells. There was no significant mitotic activity or necrosis in the tumor. On immunohistochemistry, the tumor cells were positive for CD34, vimentin, S100, EMA, and GFAP.

Nodular fasciitis, fibroosseous dysplasia, chondosarcoma and osteosarcoma should be considered in the histopathological differential diagnosis. Cartilage and hyalinized collagenous trabecules are not seen in nodular fasciitis unlike nasal chondromesenchymal hamartoma. Similarly, mature and immature cartilage islands are not seen in fibroosseous dysplasia. Chondosarcoma and osteosarcoma

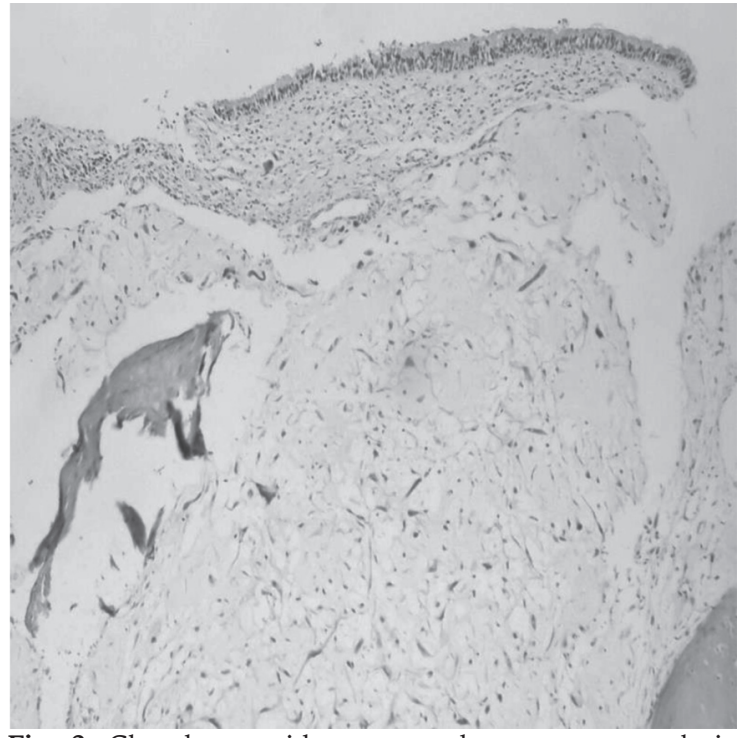

Fig. 3. Chondromyxoid stroma and osseous metaplasia beneath the ciliated respiratory epithelium (H\&E, x100)

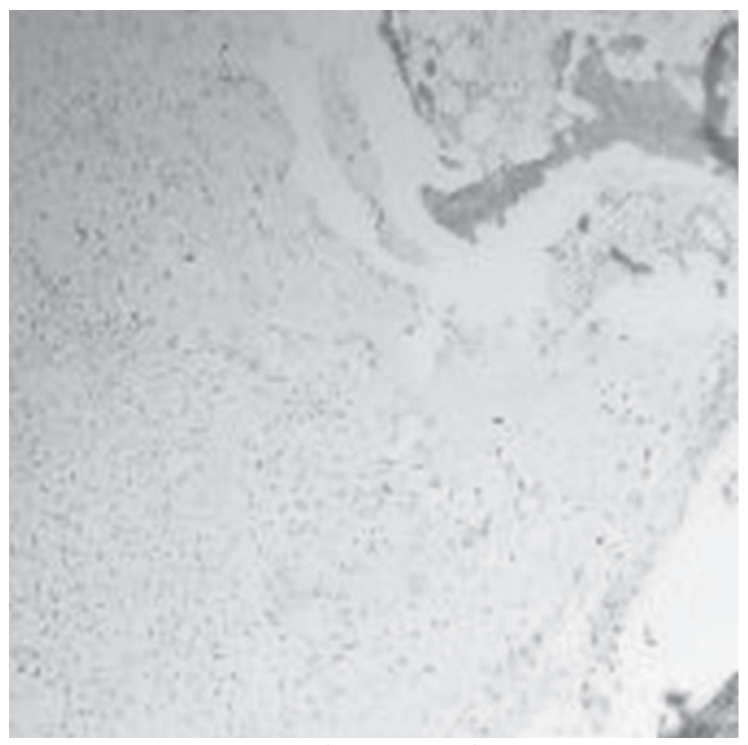

Fig. 4. Chondroid area (H\&E, x100)

were not included in differential diagnosis since there were no signs indicating malignancy. Nasopharyngeal angiofibroma, inverted papilloma, plasmoctyoma, olfactory neuroblastoma, and nasal glioma should be considered for the clinical differential diagnosis ${ }^{10}$.

Radiological imaging is directive for differential diagnosis and for evaluating surrounding tissues. Since nasal chondromesenchymal hamartoma has aggressive and infiltrative features, bony erosions can mimic malignancies. 
However, different from malignant tumors, bony destructions with sharp edges are not seen in nasal chondromesenchymal hamartoma ${ }^{9}$. On CT scans many of the cases have calcifications ${ }^{6}$. Similarly, our case had osseous components in the lesion. The differential diagnosis on radiological imaging includes hemangioma, angiofibroma, inverted papilloma, nasal giloma, giant cell reparative granuloma, ossifying fibroma, aneurysmal bone cysts, rhabdomyosarcoma, esthesioneuroblastoma and chondrosarcoma in the pediatric population ${ }^{6}$. On MR images, marked enhancement is not seen in nasal chondromesenchymal hamartoma dissimilar to hemangioma and angiofibroma ${ }^{11}$.

The treatment of nasal chondromesenchymal hamartoma is complete surgical excision. Endoscopic surgery can be suitable when the lesion is limited to the nasal cavity. Nevertheless, because the lesion has infiltrative features, it can be difficult to have tumornegative, clear surgical margins $2,5,6$. After incomplete resections and in patients with microscopic residual tumors, recurrences have been reported ${ }^{2}$. Adjuvant therapy is not needed after complete resection. However, Shet et al. ${ }^{12}$ reported that radiotherapy and combined chemotherapy is effective in regression of residual tumor if complete resection was not possible.

Although, nasal chondromesenchymal hamartoma has been accepted as a benign lesion, Li et al. ${ }^{4}$ showed malignant transformation in a case reported in 2013. The possibility of malignant transformation should be kept in mind, and detailed histologic examination should be performed particularly in adult nasal chondromesenchymal hamartoma cases.

\section{Acknowledgements}

The author would like to thank the patient

\section{REFERENCES}

1. Cotran RS, Kumar V, Collins T. Robbins Pathologic Basis of Diseases. Philadelphia: W.B. Saunders; 2003: 251.

2. McDermott MB, Ponder TB, Dehner LP. Nasal chondromesenchymal hamartoma: an upper respiratory tract analogue of the chest wall mesenchymal hamartoma. Am J Surg Pathol 1998; 22: 425-433.

3. Odell JM, Benjamin DR. Mesenchymal hamartoma of chest wall in infancy: natural history of two cases. Pediatr Pathol 1986; 5: 135-146.

4. Li Y, Yang QX, Tian XT, Li B, Li Z. Malignant transformation of nasal chondromesenchymal hamartoma in adult: a case report and review of the literature. Histol Histopathol 2013; 28: 337-344.

5. Ozolek JA, Carrau R, Barnes EL, Hunt JL. Nasal chondromesenchymal hamartoma in older children and adults: series and immunohistochemical analysis. Arch Pathol Lab Med 2005; 129: 1444-1450.

6. Johnson C, Nagaraj U, Esguerra J, Wasdahl D, Wurzbach D. Nasal chondromesenchymal hamartoma: radiographic and histopathologic analysis of a rare pediatric tumor. Pediatr radiol 2007; 37: 101-104.

7. Bahubeshi A, Bal N, Rio Frio T, et al. Germline DICER1 mutations and familial cystic nephroma. J Med Genet 2010; 47: 863-866.

8. Stewart DR, Messinger Y, Williams GM, et al. Nasal chondromesenchymal hamartomas arise secondary to germline and somatic mutations of DICER1 in the pleuropulmonary blastoma tumor predisposition disorder. Hum Genet 2014; 133: 1443-1450.

9. Norman ES, Bergman S, Trupiano JK. Nasal chondromesenchymal hamartoma: report of a case and review of the literature. Pediatr Dev Pathol 2004; 7: 517-520.

10. Kim B, Park S-H, Min HS, Rhee JS, Wang KC. Nasal chondromesenchymal hamartoma of infancy clinically mimicking meningoencephalocele. Pediatr Neurosurg 2004; 40: 136-140.

11. Wang T, Li W, Wu X, et al. Nasal chondromesenchymal hamartoma in young children: CT and MRI findings and review of the literature. World J Surg Oncol 2014; 12: 257.

12. Shet T, Borges A, Nair C, Desai S, Mistry R. Two unusual lesions in the nasal cavity of infants--a nasal chondromesenchymal hamartoma and an aneurysmal bone cyst like lesion. More closely related than we think? Int J Pediatr Otorhinolaryngol 2004; 68: 359364. 